\title{
3D Microstructural and Microanalytical Analyses of Wark-Lovering Rims in the Allende Meteorite
}

\author{
Juliane Weber, Tarunika Ramprasad, Ken Domanik, Yao-Jen Chang and Thomas Zega \\ University of Arizona, Tucson, Arizona, United States
}

Calcium-aluminum-rich inclusions (CAIs) have radiometric age dates of $4.673 \mathrm{Ga}$ and are believed to be among the first-formed solids to have condensed in the solar system (Amelin et al., 2002; Connelly et al., 2012). Some of the refractory materials that they contain match those predicted by equilibrium thermodynamic calculations and so CAIs are also believed to have directly condensed from the solar nebula (Ebel \& Grossman, 2000; Lodders, 2003), the cloud of dust and gas that gave rise to our Sun and planetary system. The microstructure of CAIs can therefore provide a unique window into early solar system chemistry. Many CAIs are surrounded by multi-layered mineral rim sequences, called WarkLovering rims (WLRs). Several processes, including condensation, metasomatic reaction and flash heating, have been proposed as possible origins for WLRs (MacPherson et al., 1981; Macpherson \& Grossman, 1984; Davis \& MacPherson, 1994; Davis et al., 1986; Wark \& Boynton, 2001; Krot et al., 1995).

Two-dimensional compositional and microstructural information on CAIs and their respective WLRs can be provided by electron- and ion-beam analyses techniques (e.g. Krot et al., 1995; Davis et al., 1986) Based on conventional electron beam techniques, 3D data could be obtained by iterative mechanical polishing and subsequent electron-beam characterization. However, focused-ion-beam (FIB) tomography can provide site-specific 3D chemical and microstructural information with greater accuracy on CAIs and WLRs by combining serial imaging with energy dispersive X-ray spectroscopy (EDS) and electron backscattered diffraction (EBSD). Here, we present the development of such techniques on a CAI expanding on our study from 2015 (Zega, 2015). Such methods are also important for the analysis of samples to be returned by the JAXA Hayabusa2 and NASA OSIRIS-REx missions.

We identified a CAI that occurs within a petrographic thick section of the Allende CV3 chondrite (University of Arizona collection. UoA 28-4-4). The Cameca SX-100 microprobe at the Kuiper Materials Imaging and Characterization Facility (KMICF) was utilized to obtain backscattered electron images and compositional maps of the entire thick section. A CAI measuring $15 \times 5 \mathrm{~mm}$ with an elongated shape was identified in the thick section. Fine-grained melilite, pyroxene, spinel and hibonite occur throughout the CAI. In addition, secondary phases, namely nepheline and sodalite occur within the WLR's and the center of the CAI. The CAI is surrounded by a Wark-Lovering Rim which contains hibonite, sodalite, melilite and spinel.

To develop 3D FIB tomography, we selected regions of interest (ROI) based on mineral assemblages and potential boundaries between different layers of the WLR structure. Thus, after the ROI was chosen, we setup automated slice-and view measurements integrating secondary electron images and quasibackscattered images using an integrated mirror detector. The slice thickness was set to $10 \mathrm{~nm}$ in order to balance acquisition time and sampling rate. For different tomography measurements, the probed volume was between 259 and $1500 \mu \mathrm{m}^{3}$ due to gradually developing best measurement parameters We collected between 319 and 1137 slices, respectively. 
For this work we reconstruct an interface between $\mathrm{MgAl}_{2} \mathrm{O}_{4}$ (spinel) and $\mathrm{Ca}_{2} \mathrm{Al}_{2} \mathrm{SiO}_{7}$ (melilite). We acquired secondary images using the ThermoFisher Helios 660 FIB-SEM instrument at KMICF. We find porosity present within the grains and at the grain boundary. We will discuss the specific challenges of preparing fine-grained meteorites for EBSD and tomography analyses.

\section{References}

Amelin, Y., Krot, A. N., Hutcheon, I. D. \& Ulyanov, A. A. (2002). Lead isotopic ages of chondrules and calcium-aluminum-rich inclusions. Science 297, 1678-1683.

Connelly, J. N., Bizzarro, M., Krot, A. N., Nordlund, Å., Wielandt, D. \& Ivanova, M. A. (2012). The absolute chronology and thermal processing of solids in the solar protoplanetary disk. Science 338, 651655.

Davis, A. M. \& MacPherson, G. J. (1994). Constraints on the formation of rims on CAIs. Meteoritics 29, 458-459.

Davis, A. M., MacPherson, G. J. \& Hinton, R. W. (1986). Rims revealed-ion microprobe analysis of individual rim layers in a Vigarano type A inclusion. Meteoritics 21, 349.

Ebel, D. S. \& Grossman, L. (2000). Condensation in dust-enriched systems. Geochimica et Cosmochimica Acta 64, 339-366.

Krot, A. N., Scott, E. R. D. \& Zolensky, M. E. (1995). Mineralogical and chemical modification of components in CV3 chondrites: Nebular or asteroidal processing? Meteoritics 30, 748-775.

Lodders, K. (2003). Solar system abundances and condensation temperatures of the elements. The Astrophysical Journal 591, 1220.

Macpherson, G. J. \& Grossman, L. (1984). "Fluffy" Type A Ca-, Al-rich inclusions in the Allende meteorite. Geochimica et Cosmochimica Acta 48, 29-46.

MacPherson, G. J., Grossman, L., Beckett, J. R. \& Allen, J. M. (1981). Origin of rims on coarse-grained inclusions in the Allende meteorite. In Lunar and Planetary Science Conferencevol. 12, pp. 648-651.

Wark, D. \& Boynton, W. V (2001). The formation of rims on calcium-aluminum-rich inclusions: Step IFlash heating. Meteoritics \& Planetary Science 36, 1135-1166.

Zega, T. J. (2015). The First Solar System Solids as Revealed Through Slice-and-View Imaging. Microscopy and Microanalysis 21, 2105-2106. 